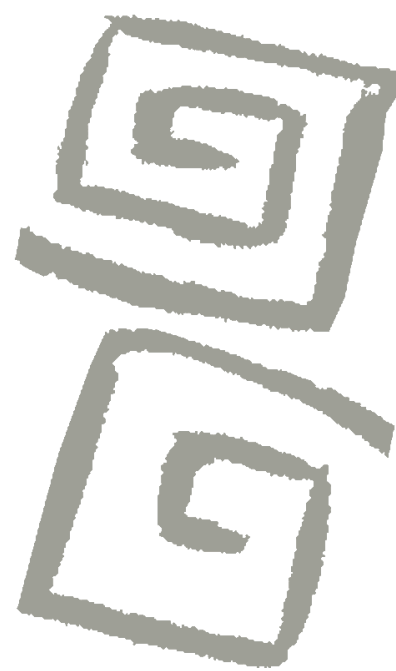

\title{
Factores asociados a la ocurrencia de tuberculosis y su distribución espacial en una ciudad brasileña, 1991-2010
}

\author{
Factors associated with the occurrence of \\ tuberculosis and its spatial distribution in a Brazilian \\ city, 1991-2010
}

${ }^{1}$ Doctora en Salud Colectiva. Instituto Aggeu Magalhães, Fiocruz Pernambuco. Pernambuco, Brasil. $\triangle$ iD

${ }^{2}$ Doctor en Salud Coletiva. Docente, Instituto Aggeu Magalhães, Fiocruz Pernambuco. Recife, Pernambuco, Brasil. $\triangle$ iD

${ }^{3}$ Doctora en Salud Coletiva. Docente, Instituto Aggeu Magalhães, Fiocruz Pernambuco. Recife, Pernambuco, Brasil. $\bowtie$ iD
Amanda Priscila de Santana Cabral Silva', Wayner Vieira de Souza², Maria de Fátima Pessoa Militão de Albuquerque ${ }^{3}$

RESUMEN Se buscó identificar los factores asociados a la incidencia de tuberculosis y a la distribución espacial de la enfermedad en Olinda, Pernambuco, entre 1991 y 2010. Para el estudio de los factores asociados a la enfermedad se aplicó la regresión de Poisson y se utilizó la razón de morbilidad estandarizada para el análisis exploratorio espacial. Si bien se observó una reducción en la incidencia media de tuberculosis en Olinda, la tasa se mantiene alta comparada con la media nacional. El mapeo por quinquenios sugiere aumentos hasta 2005, con una caída entre 2006 y 2010, además de la persistencia de alta incidencia en áreas con mayores necesidades socioeconómicas. Las mayores incidencias de tuberculosis estuvieron asociadas a la proporción de analfabetos, de jefes de familia sin ingresos, de domicilios sin abastecimiento de agua, de adultos mayores, a la presencia de más de un caso de retratamiento en el sector censal, y a los domicilios con más de un caso nuevo de tuberculosis. Se concluye que es necesario incorporar el componente espacial a la organización de los servicios de salud y la planificación de la vigilancia epidemiológica de la tuberculosis.

PALABRAS CLAVES Mycobacterium Tuberculosis; Vigilancia Epidemiológica; Epidemiología; Análisis Espacial; Factores Socioeconómicas; Brasil.

ABSTRACT This article seeks to identify factors associated with the incidence of tuberculosis and the spatial distribution of the disease in Olinda, Pernambuco, from 1991 to 2010. In order to study the factors associated with the disease, Poisson regression was applied and standardized morbidity ratios were utilized for the spatial exploratory analysis. Although a reduction in the average incidence of tuberculosis in Olinda was observed, the rate remains high in comparison with the national average. Mapping according to five-year periods suggests rate increases until 2005, with a decline between 2006 and 2010 and the persistence of high incidence in areas of greater socioeconomic need. The highest tuberculosis incidence rates were associated in each area with the proportions of illiteracy, of heads of household without income, of households lacking water supply and of older adults, as well as with the presence of cases of retreatment and of households with two or more new cases of tuberculosis. Incorporating a spatial component is key for the organization of health services and the planning of epidemiological surveillance for tuberculosis.

KEY WORDS Mycobacterium Tuberculosis; Epidemiological Surveillance; Epidemiology; Spatial Analysis; Socioeconomic Factors; Brazil. 


\section{INTRODUCCIÓN}

Las tasas de morbimortalidad de tuberculosis en Brasil, entre los años 1990 y 2010, muestran una tendencia de reducción cercana al $50,0 \%$ en la incidencia y del $70,0 \%$ en la mortalidad ${ }^{(1)}$. A pesar de la disminución de los indicadores, sigue siendo un reto el número de casos y defunciones por la enfermedad en el país, que aún integra el grupo de los 22 países que concentran el $80 \%$ de los casos de tuberculosis en el mundo. En 2013, Brasil ocupó la posición 16 en números absolutos de $\operatorname{casos}^{(2)}$, año en que se notificaron 73.000 casos nuevos (33,5 casos por 100.000 habitantes) y 4.577 defunciones $(3,2 \text { defunciones por } 100.000 \text { habitantes })^{(2,3)}$. Ser de sexo masculino, adulto joven ( 25 a 49 años), con estado civil soltero, divorciado o viudo, o vivir solo son algunas de las características asociadas a las mayores incidencias de tuberculosis. Una mayor movilidad espacial de estos individuos, un menor apoyo familiar, que aumenta la vulnerabilidad en momentos de estrés $\mathrm{O}$, incluso, cuestiones de orden biológico -en el caso del sexo masculino- que lo hacen más susceptibles a las infecciones del tracto respiratorio, son algunas de las posibles explicaciones de estas interrelaciones $\mathrm{s}^{(4,5,6,7)}$.

Desde otra perspectiva, los adultos mayores han ganado protagonismo en la endemia en el país, por ser más vulnerables a la reactivación endógena y reinfección exógena. La incidencia de la enfermedad se ha desplazado a la franja etaria más avanzada y se espera que, en algunas décadas, los casos asociados con sida se reduzcan progresivamente y aumenten los casos de reactivación en adultos mayores ${ }^{(5,7,8)}$.

Ciertas regiones caracterizadas por la alta proporción de jefes de familia con bajos niveles de ingresos y/o de escolaridad son reflejo de un conjunto de condiciones socioeconómicas precarias, que pueden aumentar la vulnerabilidad a la tuberculosis y comprometer el acceso a la información, al conocimiento y a los servicios de salud, además de perjudicar la adhesión al tratamiento ${ }^{(6,9,10,11)}$.
Muchas investigaciones adoptan la proporción de domicilios beneficiados con abastecimiento de agua, recolección de residuos y cloacas, dado que esas características pueden servir como un proxy de la situación socioeconómica de la población en estudio. En conjunto con otros factores, estos atributos pueden explicar la ocurrencia de una mayor incidencia, prevalencia o desenlaces desfavorables, como abandono o fallecimiento por tuberculosis ${ }^{(12,13,14)}$.

Factores individuales y colectivos pueden influir en un proceso desigual de transmisión de la tuberculosis en la población, siendo uno de los retratos más significativos de la inequidad social en salud. La relación entre condición de vida y la persistencia de la tuberculosis es ampliamente descripta, lo cual no significa que este abordaje esté agotado. Conocer la características locales que influyen en la variación de la incidencia del evento, podrá impactar mucho más en la carga de la enfermedad que detenerse, exclusivamente, a identificar y rectificar fragilidades en los programas de control ${ }^{(15)}$.

Cada lugar puede ser considerado como un producto de la acumulación histórica, ambiental y social, capaz de propiciar la ocurrencia y la persistencia de enfermedades. De esta forma, el lugar es una muestra de la situación de salud de la población que lo habita ${ }^{(16)}$. Ante el complejo cuadro sanitario en el cual se inserta la tuberculosis, se torna fundamental la superación de las formas tradicionales de análisis utilizadas por la epidemiología.

Desde la década de 1970, un movimiento crítico en el sector salud destacó la fragilidad de la ciencia epidemiológica, al ser utilizada de forma aislada, para el entendimiento del proceso salud-enfermedad a luz del proceso de urbanización, de la intensificación de las relaciones sociales, del surgimiento de nuevas enfermedades y de los impactos ambientales y su relación con la salud(17). La sugerencia fue incorporar nuevas técnicas de análisis y hasta incluso otras categorías de análisis de otras ciencias como la economía, la sociología y la geografía. 
En el mismo período, la renovación del pensamiento geográfico liderada por Milton Santos trajo la posibilidad de estrechar las relaciones entre la geografía y la salud. El espacio, según la definición miltoniana, está formado por dos componentes que interactúan continuamente: la configuración territorial y la dinámica social. La primera es producto de la disposición de elementos naturales y artificiales (plantaciones, canales, puertos, edificios residenciales $\mathrm{o}$ comerciales, industriales); mientras que la dinámica social está dada por el conjunto de variables económicas, culturales y políticas que, dependiendo del momento histórico, dan significación a los objetos presentes en la configuración territorial ${ }^{(18)}$.

El espacio pasa a ser entendido como proceso y producto de las relaciones sociales, que se construye como instancia social. Extrapola las geometrías, y se transforma en una categoría, un escenario formado por procesos, flujos o movimientos, en que cada período de tiempo presentará nuevos contenidos y nuevas funciones ${ }^{(18,19)}$. A partir de esta perspectiva, a la epidemiología se le permite cambiar el foco de análisis centrado en la enfermedad hacia el análisis de las condiciones de su ocurrencia. Así, la ocurrencia de la enfermedad no sería solo producto de la presencia del agente etiológico, sino resultado de una dinámica social compleja ${ }^{(17)}$.

La distribución espacial y temporal de la ocurrencia de la tuberculosis puede sustentar la comprensión de su dinámica, al presentar tendencias, áreas susceptibles y asociación con las características locales, que en conjunto con la condiciones sociales y ambientales generan una estratificación de riesgos sociales de los grupos poblacionales. Este contexto tiende a delimitar diferencias del acceso y uso de los servicios de salud ${ }^{(20,21)}$.

Se han realizado diversas investigaciones con abordaje geoespacial de la tuberculosis tanto en el ámbito nacional como internacional, a partir del mapeo de la morbimortalidad de tuberculosis y el análisis de su distribución espacial con indicadores sociales, socioeconómicos o de condiciones de $\operatorname{vida}^{(8,20,22,23,24,25,26,27)}$. Sin embargo, considerando, por un lado, el fácil acceso a las bases de datos de casos de la enfermedad, a las bases cartográficas, los instrumentos de colectas de datos geográficos y softwares y, por otro, el uso, aún tímido, de esta estrategia como soporte a la planificación de las actividades de control de la enfermedad, resulta relevante explorar el potencial de su utilización en los servicios de salud, en pro de la implementación de una vigilancia de la tuberculosis de base territorial.

En el municipio de Olinda reside el $4 \%$ de la población de Pernambuco y se notifica un promedio de 300 casos de tuberculosis por año, lo que representa el $7 \%$ del total de casos del estado. Este estudio tiene por objetivo identificar los determinantes asociados a la incidencia del evento y la dinámica espacio-temporal en dos décadas (19912010) lo que podrá señalar tendencias que muestren la necesidad de optimizar o modificar las estrategias y acciones de vigilancia epidemiológica utilizadas para el control del evento en el municipio.

Esta investigación forma parte de la tesis de doctorado titulada Dos décadas de tuberculosis en una ciudad del nordeste brasileño: Avances y desafíos en el tiempo y en el espacio realizada por la autora principal de este artículo en el Instituto Aggeu Magalhães de la Fundação Oswaldo Cruz.

\section{MÉTODOS}

Se trata de un estudio ecológico llevado a cabo en el municipio de Olinda, estado de Pernambuco, nordeste de Brasil. En 2010, contaba con 377.779 habitantes distribuidos en un territorio de $40,8 \mathrm{~km}^{2}$, lo que configura una de las mayores densidades demográficas del país, con 9.259 habitantes $/ \mathrm{km}^{2}$.

El municipio puede ser dividido en áreas relativamente homogéneas en cuanto a sus patrones habitacionales. En la región este, con mejor infraestructura urbana, es donde reside la población de ingresos medios y altos; el área central posee conjuntos habitacionales con un irregular abastecimiento de 
agua y baja cobertura de cloacas; y el área oeste está compuesta por patrones habitacionales medio e inferior, incluyendo las favelas y asentamientos resultantes de la ocupación legalizada de áreas de poco valor inmobiliario, como laderas y cerros, con una infraestructura urbana precaria ${ }^{(28)}$.

El período de estudio comprende desde el 1 de enero de 1991 al 31 de diciembre de 2010 y la población de estudio estuvo conformada por los casos nuevos de tuberculosis residentes en Olinda notificados en el Sistema de Información de Eventos de Notificación (SINAN).

Con el objetivo de identificar la tendencia de la tuberculosis en el municipio, para cada uno de los cuatro quinquenios (1991-1995, 1996-2000, 2001-2005 y 2006-2010) se calcularon las respectivas incidencias de tuberculosis, teniendo como numerador la media anual de casos nuevos notificados y, como denominador, la población promedio del período. Posteriormente, se identificaron los posibles factores asociados a la ocurrencia de la tuberculosis para cada quinquenio, y se utilizaron los sectores censales del municipio como unidad de análisis. Se adoptó el modelo de regresión de Poisson, un tipo de modelo lineal generalizado, con función de enlace logarítmica en la que las observaciones son independientes, el logaritmo de conteo varía linealmente con las variables independientes y el número de ocurrencias tiene una media y variancia iguales. En el modelo de regresión de Poisson la variable de respuesta está expresada por:

$\mathrm{y}=e^{\beta_{0}+\beta_{1} \mathrm{x}_{1}+\beta_{2} \mathrm{x}_{2}+\ldots+\beta_{k} \mathrm{x}_{k}}$

De donde:

$\log (\mathrm{y})=\beta_{0}+\beta_{1} \mathrm{x}_{1}+\beta_{2} \mathrm{x}_{2}+\ldots+\beta_{k} \mathrm{x}_{k}$

El Cuadro 1 muestra las variables socioeconómicas predictoras estudiadas, relacionadas con el programa de control de la tuberculosis. Considerando que el análisis fue realizado por quinquenio, las fuentes de las variables fueron, de 1991 a 1995, el Censo 1991; de 1996 a 2000 y de 2001 a 2005, el Censo 2000; y de 2006 a 2010, el Censo 2010. Por una cuestión de acceso y disponibilidad de datos, la variable proporción

Cuadro 1. Definición de las variables estudiadas en los modelos explicativos de la ocurrencia de la tuberculosis en Olinda, Pernambuco, Brasil. 1991-2010.

\begin{tabular}{|c|c|c|}
\hline VARIABLES & DEFINICIÓN & CATEGORIZACIÓN DEL SECTOR \\
\hline $\begin{array}{l}\text { Presencia de domicilio con más de } \\
\text { un caso nuevo en el período (a) }\end{array}$ & $\begin{array}{l}\text { Sector censal que presentó domicilio(s) con } \\
\text { dos o más casos nuevos en el quinquenio } \\
\text { estudiado }\end{array}$ & $\begin{array}{l}1=\text { Presencia de domicilio con hasta } 1 \text { caso } \\
\text { nuevo } \\
2=\text { Presencia de domicilio con } 2 \text { o más casos } \\
\text { nuevos }\end{array}$ \\
\hline $\begin{array}{l}\text { Presencia de más de un caso de } \\
\text { retratamiento en el sector censal en } \\
\text { el período }\end{array}$ & $\begin{array}{l}\text { Sector censal que presentó casos de } \\
\text { retratamiento }\end{array}$ & $\begin{array}{l}1=\text { Sin caso de retratamiento } \\
2=\text { Un caso de retratamiento } \\
3=\text { Dos o más casos de retratamiento }\end{array}$ \\
\hline Densidad domiciliaria(b) & Media de residentes por domicilio & $\begin{array}{l}\text { Cuartil 1, 2, } 3 \text { y } 4 \text { (cuartil } 1 \text { con menores } \\
\text { densidades domiciliares) }\end{array}$ \\
\hline $\begin{array}{l}\text { Proporción de jefe de familia sin } \\
\text { ingresos }\end{array}$ & $\begin{array}{l}\text { Proporción de domicilios del sector censal con } \\
\text { jefe de familia sin ingresos }\end{array}$ & $\begin{array}{l}\text { Cuartil 1, 2, } 3 \text { y } 4 \text { (cuartil } 1 \text { con menores } \\
\text { proporciones) }\end{array}$ \\
\hline Media de ingresos domiciliarios ${ }^{(b)}$ & $\begin{array}{l}\text { Media de ingresos de los domicilios del sector } \\
\text { censal }\end{array}$ & $\begin{array}{l}\text { Cuartil 1, 2, } 3 \text { y } 4 \text { (cuartil } 1 \text { con mayor media } \\
\text { de ingresos) }\end{array}$ \\
\hline Proporción de analfabetos ${ }^{(b)}$ & $\begin{array}{l}\text { Proporción de residentes del sector censal sin } \\
\text { escolaridad }\end{array}$ & $\begin{array}{l}\text { Cuartil 1, 2, } 3 \text { y } 4 \text { (cuartil } 1 \text { con menor } \\
\text { proporción) }\end{array}$ \\
\hline Proporción de adultos mayores(b) & $\begin{array}{l}\text { Proporción de residentes del sector censal } \\
\text { con } 60 \text { años o más }\end{array}$ & $\begin{array}{l}\text { Cuartil 1, 2, } 3 \text { y } 4 \text { (cuartil } 1 \text { con menor } \\
\text { proporción) }\end{array}$ \\
\hline $\begin{array}{l}\text { Proporción de domicilios sin } \\
\text { abastecimiento de agua }^{(b)}\end{array}$ & $\begin{array}{l}\text { Proporción de domicilios del sector censal sin } \\
\text { abastecimiento de agua }\end{array}$ & $\begin{array}{l}\text { Cuartil 1, 2, } 3 \text { y } 4 \text { (cuartil } 1 \text { con menor } \\
\text { proporción) }\end{array}$ \\
\hline
\end{tabular}

Fuente: Elaboración propia basada en el Sistema de Información de Eventos de Notificación (a) y en el Instituto Brasileño de Geografía y Estadística (b) $(1991,2000,2010)$. 
de adultos mayores no integró del modelo inicial de análisis de regresión en el primer quinquenio, siendo examinada solo en los quinquenios siguientes.

Las variables relacionadas con el programa de control tuvieron categorías fijas en los cuatro quinquenios. Considerando el período analizado y las diferentes fuentes censales utilizadas, las variables relacionadas con condiciones socioeconómicas fueron categorizadas en cuartiles. También se adicionó al modelo de regresión la población residente en cada sector censal, como variable de exposición.

A partir de los residuos de la regresión se infirió, para cada quinquenio, la presencia de autocorrelación espacial por medio del índice de Moran global. Los índices calculados dieron valores inferiores al 20\%, lo que justifica no adicionar el factor espacial al modelo de regresión. Aunque tres de los cuatro quinquenios haya presentado un índice de Moran global con significancia estadística igual o mayor que $99 \%$, ese resultado puede ser justificado por el gran número de observaciones, que están representados por los sectores censales analizados.

Para la selección de las variables que componen el modelo final de riesgo, se aplicó el método de selección por eliminación retroactiva, que consiste en eliminar las variables predictoras, una a una, en cada interacción, a partir del modelo completo, hasta que se obtenga un conjunto de variables que genere el modelo con mejor ajuste. Se utilizó el criterio de información de Akaike, una medida de la calidad del ajuste del modelo basada en el número de parámetros y en la máxima verosimilitud entre los valores reales y los valores previstos de la variable resultado ${ }^{(29)}$. El análisis se realizó con el software Stata 12.0.

Para realizar el análisis espacial se necesitaban datos georreferenciados. Para los casos notificados entre 1991 y 2000 se accedió a datos georreferenciados originalmente por sectores censales ${ }^{(30,31)}$. Para el período 2001-2010, los casos se georreferenciaron en un GPS y se geocodificaron por medio del GPSVisualizer y Google
Earth por el Núcleo de Geoprocesamiento de la Secretaria de Salud de Olinda y por la Fiocruz Pernambuco. La trama digital de los sectores censales se tomó de una investigación anterior ${ }^{(30)}$ y de las bases de los censos demográficos IBGE 2000 y $2010^{(32,33)}$.

Para el análisis espacial de la tuberculosis se adoptaron, como unidad de análisis, los sectores censales de residencia de los casos nuevos, según quinquenio de ocurrencia. El análisis espacial descriptivo fue realizado a través de la distribución de la tasa media anual de la incidencia de tuberculosis para cada quinquenio, según sector censal de residencia. Se tomó como numerador el total de casos de tuberculosis en cada quinquenio dividido por cinco y, como denominador, la población de cada sector promedio del período, de acuerdo con el censo demográfico de referencia. La incidencia media anual de la enfermedad en Brasil en la última década estudiada fue de aproximadamente 40 casos por 100.000 habitantes $^{(3)}$. Esa tasa fue la utilizada como parámetro para las categorías de los mapas temáticos presentados:

a. $<40$ casos por 100.000 habitantes: incidencia inferior a la media nacional.

b.40-60 casos por 100.000 habitantes: incidencia superior a la media nacional en hasta $50 \%$.

c. $60-80$ casos por 100.000 habitantes: incidencia superior a la media nacional, de 50 a $100 \%$.

d. 80-100 casos por 100.000 habitantes: incidencia superior a la media nacional, de 100 a $150 \%$.

e. $>100$ casos por 100.000 habitantes: incidencia superior a la media nacional, en más de $150 \%$.

Para el análisis exploratorio se calculó la razón de morbilidad estandarizada. El objetivo fue verificar la hipótesis de que la incidencia en el tiempo y en el espacio no tuviera variación, para lo cual se adoptó como numerador la incidencia observada en el sector y como denominador la incidencia esperada, en este caso, la incidencia media en Olinda durante las dos décadas, lo que se esperaría 
que ocurriera ante la ausencia de exposición, si esta no afectara la distribución en el tiempo y en el espacio persona-tiempo ${ }^{(34)}$. Así, la razón de morbilidad estandarizada $=$ incidencia observada / incidencia esperada, constituye una medida de riesgo.

\section{Consideraciones éticas}

El proyecto de investigación fue sometido al Comité de Ética en Investigación del Centro de Pesquisas Aggeu Magalhães, y aprobado con el CAAE N³3878514.4.0000.5190; parecer 782.312/2014.

\section{RESULTADOS}

Entre 1991 y 2010, se notificaron 6.202 casos nuevos de tuberculosis, un promedio de 310 casos nuevos por año u 86 casos por 100.000 habitantes. Las tasas de incidencias por quinquenio (Tabla 1 ) presentaron una tendencia a la caída $\left(\chi^{2}=14,2 ; p=0,0002\right)$.

Las mayores ocurrencias de tuberculosis, en todos los quinquenios, se asociaron a residir en sectores con domicilios con más de un caso nuevo, alta proporción de analfabetismo y alta proporción de adultos mayores, característica este última que fue testada a partir del segundo quinquenio, entre 1996 y 2000. En la primera década la densidad domiciliaria presentó una magnitud que no deja clara su relación con la ocurrencia de la enfermedad. Por una cuestión metodológica la variable fue mantenida, dado que el criterio de información de Akaike indicó un mejor ajuste para los modelos cuando este componente estuvo presente.

Entre 1991 y 1995, también se mantuvieron en el modelo residir en sectores con domicilios con jefe de familia sin ingresos y sin abastecimiento de agua (Tabla 2). El modelo final propuesto en este quinquenio explicó apenas el $8 \%$ de la ocurrencia de tuberculosis en el municipio, con poco poder explicativo posiblemente resultante de la calidad de los datos disponibles en el período analizado.
Tabla 1. Distribución del promedio de casos, población y tasa de incidencia (por 100.000 habitantes) por tuberculosis, según quinquenio de ocurrencia. Olinda, Pernambuco, Brasil. 1991-2010.

\begin{tabular}{cccc} 
QUINQUENIO & $\begin{array}{c}\text { PROMEDIO DE } \\
\text { CASOS NUEVOS }\end{array}$ & $\begin{array}{c}\text { PROMEDIO DE } \\
\text { POBLACIÓN }\end{array}$ & INCIDENCIA \\
\hline $1991-1995$ & 329 & 351.451 & 93,7 \\
$1996-2000$ & 329 & 353.046 & 93,3 \\
$2001-2005$ & 299 & 376.066 & 79,5 \\
$2006-2010$ & 283 & 394.850 & 71,6
\end{tabular}

Fuente: Elaboración propia basada en el Sistema de Información de Eventos de Notificación y en el Instituto Brasileño de Geografía y Estadística.

En el segundo quinquenio, se mantuvieron en el modelo las dos variables relacionadas al Programa de Control de Tuberculosis: sectores con domicilio con más de un caso nuevo y sectores con dos o más casos de retratamiento, además de regiones con mayor proporción de adultos mayores, domicilios con jefe de familia sin ingresos y analfabetismo (Tabla 2). En este período, el modelo final fue capaz de explicar el $54 \%$ de la ocurrencia de la tuberculosis en el municipio.

Entre 2001 y 2005 el modelo propuesto contempla las variables sector con más de un caso nuevo, sector con más de un caso de retratamiento, proporción de adultos mayores, analfabetismo y presencia de domicilios sin abastecimiento de agua (Tabla 3). En el último quinquenio, el modelo final propuesto incluye residir en sectores con más de un caso nuevo y tener más de un caso de retratamiento, además de baja escolaridad, mayor proporción de adultos mayores y mayor proporción de jefes de familia sin ingresos (Tabla 3 ).

El análisis espacial descriptivo (Figura 1) de la distribución de la incidencia reveló que, en los dos primeros quinquenios, más del $45 \%$ de los sectores presentó una incidencia que superó el doble de la media nacional. De 2001 a 2005 ese porcentaje llegó al 27,4\%, reducción similar a la incidencia media del municipio. Sin embargo, entre 2006 y 2010 en aproximadamente el $60 \%$ de los sectores censales aún persistía una incidencia superior a la media nacional. 
Tabla 2. Factores asociados que componen los modelos finales de riesgo de la ocurrencia de la tuberculosis. Olinda, Pernambuco, Brasil. 1991-2000.

FACTORES ASOCIADOS
1991 a 1995
Sector con domicilio con más de 1 caso nuevo
Hasta 1 caso nuevo
2 casos nuevos o más
Densidad domiciliaria
$<4,1$
$4,1-4,4$
$4,4-4,7$
$4,7-5,3$

\section{RR}

Porcentaje de domicilios del sector con jefe de familia sin ingresos

$<3,4$

$$
3,4-6,8
$$

$6,8-11,0$

$11,0-53,5$

Porcentaje de residentes analfabetos en el sector

$<5,0$

$5,0-13,7$

$13,7-23,0$

$23,0-55,2$

Porcentaje de domicilios del sector sin abastecimiento de agua

$<0,33$

$0,33-10,8$

$10,8-38,0$

$38,0-99,1$

1996 a 2000

Sector con domicilio con más de 1 caso nuevo

Hasta 1 caso nuevo

2 casos nuevos o más

Densidad domiciliaria

$<3,72$

$3,72-3,93$

$3,93-4,09$

$4,09-4,70$

Porcentaje de domicilios del sector con jefe de familia sin ingresos

$$
<5,7
$$

$5,7-10,8$

$10,8-15,9$

$15,9-47,9$

Porcentaje de residentes analfabetos en el sector

$$
<3,3
$$

$$
3,3-10,9
$$

$10,9-19,9$

$19,9-37,8$

Porcentaje de adultos mayores

$<6,6$

$6,6-8,6$

$8,6-10,7$

$10,7-21,0$

Presencia de 1 o más casos de retratamiento en el sector

Sin casos

1 caso

20 más casos

Fuente: Elaboración propia basada en el Sistema de Información de Eventos de Notificación y en el Instituto Brasileño de Geografía y Estadística. RR = Riesgo relativo. IC 95\% = Intervalo de confianza del 95\%. Criterio de Información de Akaike (AIC)

1991 a $1995=$ AIC modelo inicial $=1631,179-$ AIC modelo final $=1630,738 ; R^{2}=0,0814$

1996 a $2000=$ AIC modelo inicial $=1511,604-$ AIC modelo final = 1452,400; $R^{2}=0,5412$

$\begin{array}{ll}0,85-1,39 & 0,48 \\ 0,67-1,23 & 0,53\end{array}$

$0,88-1,59 \quad 0,26$

$0,85-1,67$

$0,96-2,30 \quad 0,08$

$0,75-2,33 \quad 0,33$

0,89-1,77 0,18

0,63-1,42 $\quad 0,80$

$0,75-1,80 \quad 0,50$

$1,39-1,82 \quad 0,00$

$0,99-1,46 \quad 0,06$

$0,77-1,19 \quad 0,72$

$0,75-1,13 \quad 0,46$

$1,03-1,58 \quad 0,02$

0,92-1,36 $\quad 0,24$

1,17-1,83 $\quad 0,001$

$1,08-1,81 \quad 0,01$

1,00 -

$\begin{array}{lll}1,43 & 1,18-1,73 & 0,000 \\ 1,40 & 1,15-1,71 & 0,001 \\ 1,36 & 1,10-1,68 & 0,004\end{array}$

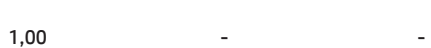

$1,48 \quad 1,24-1,78 \quad 0,00$

$\begin{array}{lll}1,48 & 1,24-1,78 & 0,00\end{array}$


Tabla 3. Factores asociados que componen los modelos finales de riesgo de la ocurrencia de la tuberculosis. Olinda, Pernambuco, Brasil. 2001-2010.

\begin{tabular}{|c|c|c|c|}
\hline FACTORES ASOCIADOS & RR & IC $95 \%$ & Valor de $p$ \\
\hline \multicolumn{4}{|l|}{2001 a 2005} \\
\hline \multicolumn{4}{|c|}{ Sector con domicilio con más de 1 caso nuevo } \\
\hline Hasta 1 caso nuevo & 1,00 & - & - \\
\hline 2 casos nuevos o más & 2,06 & $1,56-2,73$ & $<0,001$ \\
\hline \multicolumn{4}{|c|}{ Porcentaje de residentes analfabetos en el sector } \\
\hline$<3,3$ & 1,00 & - & - \\
\hline $3,3-10,9$ & 1,50 & $1,21-1,87$ & $<0,001$ \\
\hline $10,9-19,9$ & 1,58 & $1,24-2,02$ & $<0,001$ \\
\hline $19,9-37,8$ & 1,48 & $1,12-1,95$ & 0,006 \\
\hline \multicolumn{4}{|c|}{ Porcentaje de domicilios del sector sin abastecimiento de agua } \\
\hline$<0,54$ & 1,00 & - & - \\
\hline $0,54-2,15$ & 0,80 & $0,65-0,98$ & 0,03 \\
\hline $2,15-6,57$ & 0,88 & $0,72-1,09$ & 0,24 \\
\hline $6,57-85,2$ & 0,82 & $0,66-1,01$ & 0,07 \\
\hline \multicolumn{4}{|l|}{ Porcentaje adultos mayores } \\
\hline$<6,6$ & 1,00 & - & - \\
\hline $6,6-8,6$ & 1,17 & $0,94-1,45$ & 0,166 \\
\hline $8,6-10,7$ & 1,14 & $0,90-1,45$ & 0,285 \\
\hline $10,7-21,0$ & 1,30 & $1,02-1,67$ & 0,035 \\
\hline \multicolumn{4}{|c|}{ Sector con más de un caso de retratamiento } \\
\hline Sin casos & 1,00 & - & - \\
\hline 1 caso & 1,09 & $0,92-1,29$ & 0,33 \\
\hline 20 más casos & 1,21 & $0,97-1,53$ & 0,09 \\
\hline \multicolumn{4}{|l|}{2006 a 2010} \\
\hline \multicolumn{4}{|c|}{ Sector con domicilio con más de 1 caso nuevo } \\
\hline Hasta 1 caso nuevo & 1,00 & - & - \\
\hline 2 casos nuevos o más & 2,03 & $1,63-2,52$ & $<0,001$ \\
\hline \multicolumn{4}{|c|}{ Porcentaje de domicilios del sector con jefe de familia sin ingresos } \\
\hline$<7,1$ & 1,00 & - & - \\
\hline $7,1-11,6$ & 1,32 & $1,06-1,64$ & 0,01 \\
\hline $11,6-20,6$ & 1,27 & $1,01-1,60$ & 0,04 \\
\hline $20,6-64,7$ & 1,31 & $1,03-1,67$ & 0,03 \\
\hline \multicolumn{4}{|c|}{ Porcentaje de residentes analfabetos en el sector } \\
\hline$<2,7$ & 1,00 & - & - \\
\hline $2,7-7,5$ & 1,26 & $0,99-1,59$ & 0,05 \\
\hline $7,5-14,6$ & 1,42 & $1,07-1,86$ & 0,01 \\
\hline $14,6-37,1$ & 1,25 & $0,92-1,69$ & 0,15 \\
\hline \multicolumn{4}{|c|}{ Porcentaje de adultos mayores } \\
\hline$<8,1$ & 1,00 & - & - \\
\hline $8,1-10,9$ & 1,27 & $1,03-1,58$ & 0,03 \\
\hline $10,9-14,6$ & 1,27 & $0,99-1,63$ & 0,06 \\
\hline $14,6-28,6$ & 1,37 & $1,00-1,88$ & 0,05 \\
\hline \multicolumn{4}{|c|}{ Sector con más de un caso de retratamiento } \\
\hline Sin casos & 1,00 & - & - \\
\hline 1 caso & 1,39 & $1,16-1,66$ & $<0,001$ \\
\hline 20 más casos & 2,32 & $1,77-3,06$ & $<0,001$ \\
\hline \multicolumn{4}{|c|}{$\begin{array}{l}\text { Fuente: Elaboración propia basada en el Sistema de Información de Eventos de Notificac } \\
\text { Estadística. } \\
\text { RR = Riesgo relativo. IC } 95 \%=\text { Intervalo de confianza del } 95 \% \text {. } \\
2001 \text { a } 2005=\text { AIC modelo inicial }=1376,902-\text { AIC modelo final }=1369,640 ; R^{2}=0,3224 \\
2006 \text { a } 2010 \text { = AIC modelo inicial }=1847,526 \text { - AIC modelo final }=1836,776 ; R^{2}=0,3750\end{array}$} \\
\hline
\end{tabular}



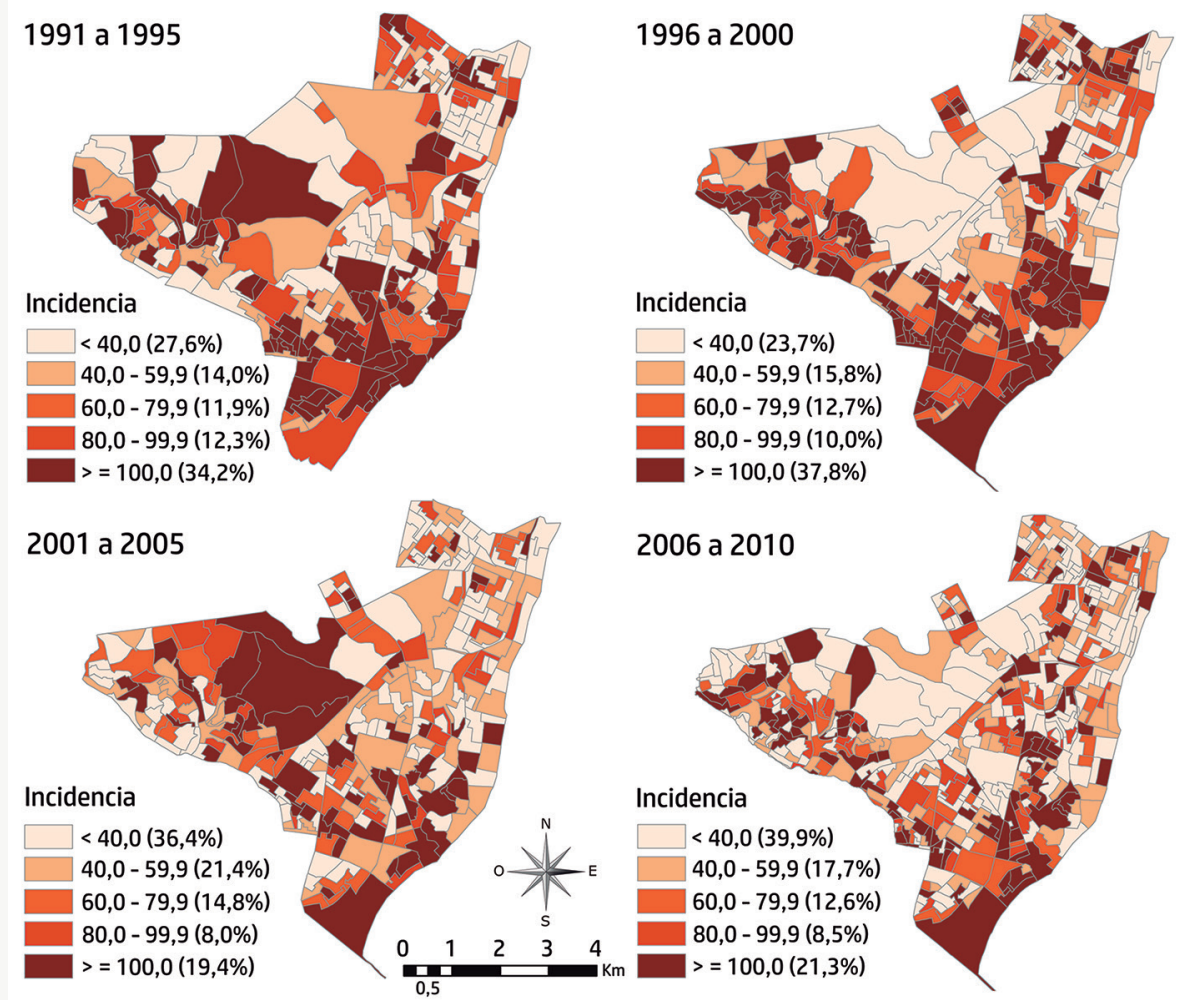

Figura 1. Incidencia de tuberculosis (por 100.000 habitantes) según sector censal de residencia. Olinda, Pernambuco, Brasil. 1991-2010.

Fuente: Elaboración propia basada en el Sistema de Información de Eventos de Notificación y en el Instituto Brasileño de Geografía y Estadística.
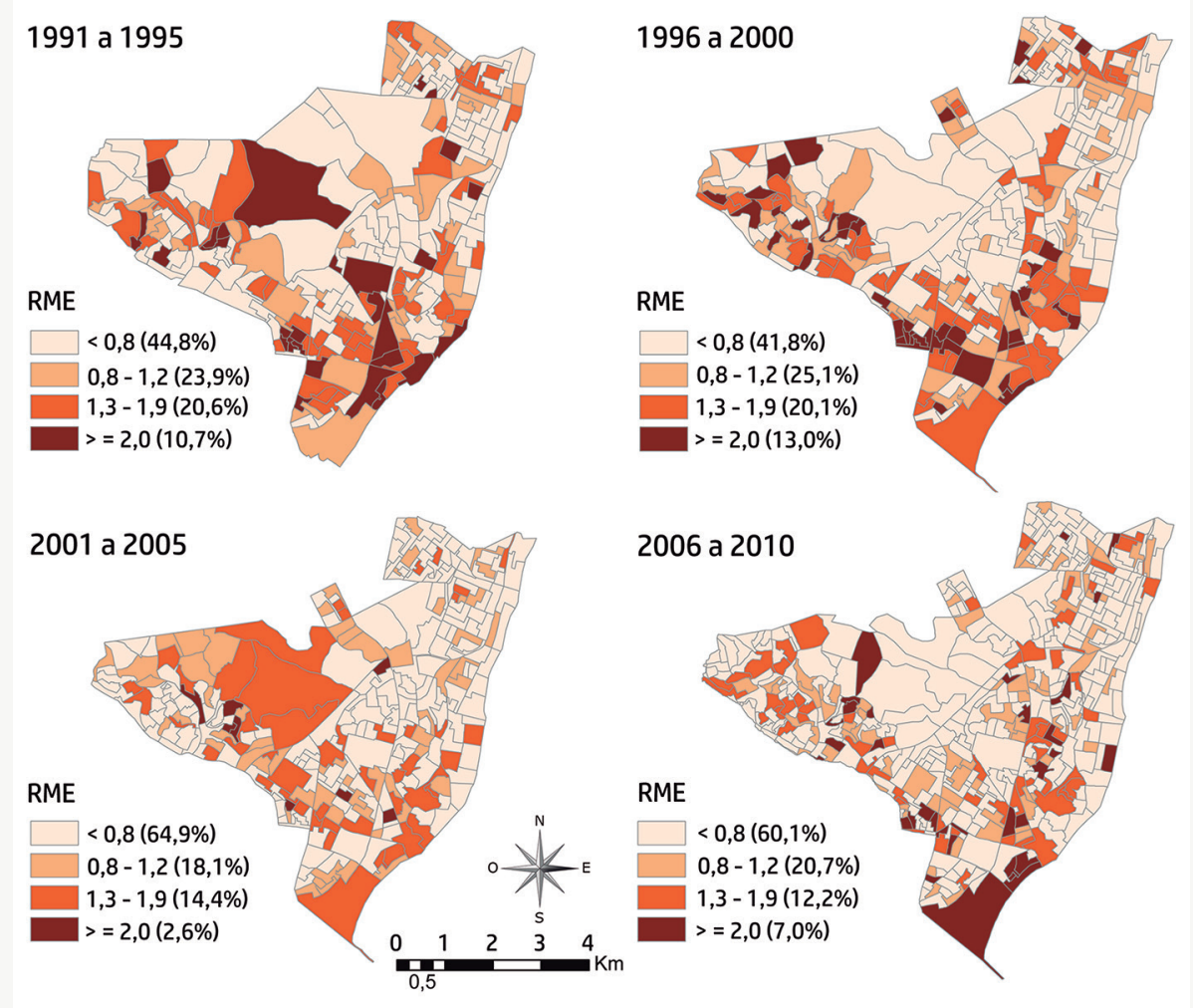

Figura 2. Distribución de la tuberculosis por sector censal de residencia, según razón de morbilidad estandarizada. Olinda, Pernambuco, Brasil. 1991-2010. 
El análisis de la razón de morbilidad estandarizada (Figura 2) revela una posible homogeneización de la enfermedad en el territorio de Olinda, pues mientras que el 31,3\% de los sectores presentaron un riesgo mayor que el $30 \%$ de ocurrencia de la tuberculosis entre 1991 y 1995, en el tercer quinquenio el $17,0 \%$ de los sectores estaba en esta condición de riesgo y solo el 2,6\% de las áreas tenía una razón de morbilidad estandarizada superior a 2,0 . Aunque se haya observado la tendencia de reducción de las tasas, en el último quinquenio, el 7\% de los sectores llegó a presentar más del doble del riesgo de la ocurrencia esperada, en una especie de recrudecimiento del problema en el tiempo y en el espacio. Aun hasta 2005 la concentración de los altos riesgos se dio en áreas al sur y al oeste del municipio mientras a partir de 2006 también se identificaron elevadas tasas al centro-este del territorio de Olinda.

\section{DISCUSIÓN Y CONCLUSIONES}

La reducción de la incidencia de la tuberculosis en Olinda acompañó la tendencia de la enfermedad en Brasil(1). A pesar de la disminución de la tasa, los números son preocupantes al compararlos con los del estado y del país. El último quinquenio, Olinda contabilizó 71,6 casos por 100.000 habitantes, que excedió el valor registrado en Pernambuco (47 casos por 100.000 habitantes) y duplicó los 35 casos por 100.000 habitantes detectados en el país ${ }^{(3,7)}$.

La bibliografía presenta una serie de determinantes y factores relacionados con la ocurrencia de tuberculosis ${ }^{(15)}$. En el presente trabajo se estudió la influencia de variables similares en el transcurso de las dos décadas analizadas. De cierta forma, esto limitó la cantidad de variables elegibles, debido a la disponibilidad de los datos y los cambios ocurridos a lo largo de los años, tanto en el censo demográfico, como en las fichas de notificación.

La regresión de Poisson fue adecuada para la identificación de las variables que conformaron los modelos explicativos de la ocurrencia de tuberculosis en el período. La categorización de las variables en cuartiles, cuando fue necesario, se mostró útil para el análisis de regresión. Se estudiaron dos variables relacionadas al Programa de Control de Tuberculosis: sectores con más de un caso de retratamiento y aquellos con domicilios con más de un caso nuevo. Durante todo el período las dos variables estuvieron asociadas a la ocurrencia de la enfermedad, excepto el retratamiento entre 1991 y 1995. Las regiones que se destacan en cuanto al retratamiento pueden reflejar el acceso y la calidad del servicio de salud prestado a esa población. También, revela una comunidad expuesta a un bacilífero por más tiempo, individuos que presentan un elevado riesgo de mal pronóstico, como la multirresistencia o la muerte ${ }^{(26,35,36)}$.

Aunque sea un dato captado en el propio banco de datos, la información sobre el domicilio con más de un caso nuevo de tuberculosis difícilmente es utilizada en la rutina del análisis de la situación de salud en las vigilancias epidemiológicas locales. Al considerar la asociación de esta variable a la ocurrencia de la enfermedad en Olinda, el presente estudio muestra la importancia del monitoreo de esa característica por parte del Programa de Control de Tuberculosis, a la luz del presupuesto de que la tuberculosis extrapola la esfera biológica, pues está asociada a las condiciones de vida y trabajo de los individuos ${ }^{(37)}$.

La complejidad de la asistencia frente a esa condición exige una organización sistematizada de la atención a esa familia. El Programa de Control de Tuberculosis podrá evaluar la posibilidad de adoptar el tratamiento supervisado, considerando su disponibilidad de recursos y el perfil de los enfermos a ser acompañados ${ }^{(37,38)}$. El profesional responsable del tratamiento supervisado en el domicilio debe disponer de conocimientos, actitudes y prácticas adecuadas para realizar el plan de actividades en el domicilio de los enfermos/familias. El contacto directo del profesional de salud con la realidad intradomiciliaria posibilita la identificación de problemas con características y soluciones peculiares. 
La transmisión intradomiciliaria de la tuberculosis es factible, como ya fue evidenciado en otros estudios ${ }^{(39,40,41)}$. Por este motivo, el Ministerio de Salud recomienda que, al descubrir un caso de tuberculosis, todos los contactos intradomiciliarios sean investigados, a fin de descartar la enfermedad ${ }^{(41)}$.

Una de las variables más adecuadas para estimar la transmisión intradomiciliaria es la media de habitantes por dormitorio, que ya fue mencionada por el propio municipio de Olinda, al inicio de la década de 1990, como asociado al mayor riesgo de ocurrencia de tuberculosis $^{(31)}$. Sin embargo, a partir del Censo 2000 del Instituto Brasileño de Geografía y Estadística (IBGE), esta variable dejó de estar disponible, y solo es posible estimar el número de habitantes por domicilio, condición que fue adoptada en este estudio. La débil asociación aquí encontrada sugiere que el dato a nivel de domicilio es menos sensible comparado con la variable relacionada con el dormitorio.

La susceptibilidad de los adultos mayores a la tuberculosis puede estar influenciada por comorbilidades y por el uso de medicamentos con acción inmunosupresora, que ya posee inmunosenescencia relacionada con la edad. Otro aspecto es el abandono del tratamiento, común en esta franja etaria, y que puede repercutir en un mal pronóstico ${ }^{(42,43)}$. La variable se mantuvo en todos los modelos finales y llegó a representar, en este grupo, un riesgo en torno al $40 \%$ mayor de ocurrencia de la tuberculosis. Estos hallazgos refuerzan cuán necesario es estar atento a este grupo etario, además de la integración inter e intra sector salud, en especial con el programa de salud del adulto mayor.

Durante las dos décadas, residir en regiones con mayor proporción de analfabetos estuvo asociado a mayores tasas de tuberculosis, y en uno de los períodos llegó a presentar un riesgo de ocurrencia 50\% mayor en esas áreas. En tres de los cuatro quinquenios los domicilios con jefe de familia sin ingresos fueron una característica presente en los modelos de riesgo para la ocurrencia de la tuberculosis. La combinación de bajos ingresos y baja escolaridad, asociada además a la cuestión etaria ya mencionada, refleja un posible acceso desigual a la información, a los bienes de consumo y a los servicios de salud $^{(11,44,45)}$.

Un estudio en una región de la India(46) alerta que la baja escolaridad puede afectar hasta incluso la entrada del caso sospechoso al sistema, dado que el individuo puede no percibirse como un caso sospechoso o no comprender el modo de transmisión de la enfermedad. Esa percepción individual es importante, considerando que, si bien el sistema de vigilancia es preferentemente de búsqueda activa, en la práctica se da de forma pasiva, con la llegada del enfermo al servicio de salud. Conocer las regiones con mayor proporción de analfabetos contribuye al desarrollo de estrategias de trabajo para proveer el acceso universal a los cuidados de tuberculosis, especialmente, entre esa población socialmente vulnerable.

El componente sanitario en este estudio fue representado por la proporción de domicilios sin abastecimiento de agua. Una mayor ocurrencia de la tuberculosis relacionada con esa variable fue observada en el primer quinquenio, de forma similar a los hallazgos de Dye ${ }^{(12)}$ cuando analizó esta relación en 134 países. Curiosamente, entre 2001 y 2005, la ausencia del abastecimiento se mantuvo en el modelo como un factor de protección. El primer quinquenio fue analizado a la luz del Censo 1991, mientras el tercer quinquenio se basó en el Censo 2000. Esa variable puede haber perdido su poder discriminatorio con el pasar de los años, dado que en el primer quinquenio esa característica era común en regiones con peor infraestructura. Al final de la década de 1990, la región del litoral del municipio pasó por una expansión inmobiliaria, con inmuebles no necesariamente ligados a la red general de abastecimiento de agua, pero con pozos propios.

Vale resaltar que, en este estudio, la mayoría de las variables que conformaron los modelos explicativos de la incidencia no deben ser consideradas, exclusivamente, como factores de riesgo para la ocurrencia de tuberculosis. Los resultados también revelan las fragilidades de las políticas sociales y de 
salud pública de Olinda, que se perpetúan hace años, impidiendo la mejora de las condiciones de vida que impactarían en varios indicadores de salud, entre ellos, los relacionados con tuberculosis.

La razón de morbilidad estandarizada se mostró como una buena opción para el análisis exploratorio sobre la distribución espacio-temporal de la ocurrencia de eventos de salud, incluyendo la tuberculosis, sobre todo para los servicios de salud. Se destaca la fácil interpretación que verifica la hipótesis de distribución homogénea del evento en el tiempo y en el espacio. Operacionalmente, la tasa esperada puede ser definida por el equipo al frente del monitoreo, que puede ser estatal o nacional, o incluso una meta establecida. Aquí se adoptó la incidencia media anual para las dos décadas en el municipio.

Se observó una reducción de la incidencia de tuberculosis en Olinda en el transcurso de las dos décadas analizadas. Sin embargo, la media de la incidencia municipal, desde el punto de vista global, se mantiene alta comparada a la media nacional $y$, al estratificar la región de estudio, se percibe un gran número de sectores con incidencia superior al doble de la media del país, sobre todo en el último quinquenio.

Adicionalmente, se verifica que las tasas en áreas de mayor carencia socioeconómica se mantienen altas, lo que coloca a la enfermedad como un marcador de inequidad social en salud. Las diferentes "Olindas" que cobraron visibilidad en este estudio precisan de diferentes estrategias para el control de la enfermedad.

Los factores asociados a la ocurrencia de la enfermedad en las dos décadas analizadas engloban características externas a la esfera del sector salud y otras que son de responsabilidad del Programa de Control de Tuberculosis. Además, esa ocurrencia no se da de forma aleatoria en el espacio, sino que se pueden identificar aglomerados de mayores tasas de incidencia. Estos hallazgos refuerzan la importancia de la incorporación del componente espacio para la planificación de las acciones de vigilancia y organización de los servicios de salud.

Otra de las cuestiones del objeto de este estudio, que deben ser investigadas para una mejor comprensión de la evolución de la distribución espacial de la enfermedad, es la posible influencia de la ampliación de la atención primaria en la detección de la tuberculosis y el patrón de la coinfección tuberculosis-VIH en el territorio. Por otro lado, conocer las características de la(s) cepa(s) de la Mycobacterium tuberculosis circulantes podría explicar la persistencia de la enfermedad en determinadas regiones del municipio. 


\section{REFERENCIAS BIBLIOGRÁFICAS}

1. Guimarães RM, Lobo AP, Siqueira EA, Borges TFF, Melo SCC. Tuberculosis, HIV, and poverty: temporal trends in Brazil, the Americas, and worldwide. Jornal Brasileiro de Pneumologia. 2012;38(4):511-517.

2. World Health Organization. Global tuberculosis report 2014 [Internet]. 2014 [citado 2 may 2016]. Disponible en: https://tinyurl.com/pv8kbvb.

3. Brasil, Programa Nacional de Controle da Tuberculose. Série histórica da taxa de incidência de tuberculose: Brasil, regiões e unidades federadas de residência por ano de diagnóstico (1990 a 2014) [Internet]. Brasília; 2015 [citado 1 mar 2016]. Disponible en: https://tinyurl.com/y89zlkya.

4. Falagas ME, Mourtzoukou EG, Vardakas KZ. Sex differences in the incidence and severity of respiratory tract infections. Respiratory Medicine. 2007;101(9):1845-1863.

5. Pereira JC, Silva MR, Costa RR, Guimarães MDC, Leite ICG. Profile and follow-up of patients with tuberculosis in a priority city in Brazil. Revista de Saúde Pública. 2015;49(6):1-12.

6. Pang PT, Leung CC, Lee SS. Neighbourhood risk factors for tuberculosis in Hong Kong. The International Journal of Tuberculosis and Lung Disease. 2010;14(5):585-592.

7. Cabral Silva APS, Souza WV, Albuquerque MFPM. Two decades of tuberculosis in a city in Northeastern Brazil: advances and challenges in time and space. Revista da Sociedade Brasileira de Medicina Tropical. 2016;49(2):211-221.

8. Vendramini SHF, Santos NSGM, Santos MLSG, Chiaravalloti-Neto F, Ponce MAZ, Gazetta CE, et al. Spatial analysis of tuberculosis/HIV coinfection: its relation with socioeconomic levels in a city in south-eastern Brazil. Revista da Sociedade Brasileira de Medicina Tropical. 2010;43(5):536-541.

9. Angelo JR. (Re)produção do espaço urbano de Juiz de Fora e a distribuição espacial da tuberculose. [Dissertação de Mestrado]. Rio de Janeiro: Escola Nacional de Saúde Pública, Fundação Oswaldo Cruz; 2008.

10. Fasca SF. Tuberculose e condições de vida: uma análise do Estado do Rio de Janeiro; Brasil, 2002. [Dissertação de Mestrado]. Rio de Janeiro: Escola Nacional de Saúde Pública, Fundação Oswaldo Cruz; 2008.

11. Garrido MS, Penna ML, Perez-Porcuna TM, de Souza AB, Marreiro LS, Albuquerque BC, et al.
Factors associated with tuberculosis treatment default in an endemic area of the Brazilian Amazon: a case control-study. PLoS One. 2012;7(6):e39134.

12. Dye C, Lönnroth K, Jaramillo E, Williams BG, Raviglione $M$. Trends in tuberculosis incidence and their determinants in 134 countries. Bulletin of the World Health Organization. 2009;87(9):683691.

13. Serpa IM, Pardo CL, Hernandez RA. Um estudio ecológico sobre tuberculosis en um municipio de Cuba. Cadernos de Saúde Pública. 2003;19(5):1305-1312.

14. Silva VL, Leal MCC, Marino JG, Marques APO. Associação entre carência social e causas de morte entre idosos residentes no município de Recife, Pernambuco, Brasil. Cadernos de Saúde Pública. 2008;24(5):1013-1023.

15. San Pedro A, Oliveira RM. Tuberculosis and socioeconomic indicators: systematic review of the literature. Revista Panamericana de Salud Pública. 2013;33(4):294-301.

16. Barcellos CC, Sabroza PC, Peiter P, Rojas LI. Organização espacial, saúde e qualidade de vida: análise espacial e uso de indicadores na avaliação de situações de saúde. Informe Epidemiológico do SUS. 2002;11(3):129-138.

17. Faria RM, Bortolozzi A. Espaço, território e saúde: contribuições de Milton Santos para o tema da geografia da saúde no Brasil. Raega-O Espaço Geográfico em Análise. 2009;17:31- 41.

18. Santos M. Por uma geografia das redes. In: Santos M. A natureza do espaço: técnica e tempo; razão e emoção. São Paulo: Hucitec; 1997. p. 261-280.

19. Santos CB, Hino $P$, Cunha TN, Villa TCS, Muniz JN. Utilização de um sistema de informação geográfica para descrição dos casos de tuberculose. Boletim de Pneumologia Sanitária. 2004;12(1):7-12.

20. Queiroga RPF, Sá LD, Nogueira JA, Lima ERV, Silva ACO, Pinheiro PGOD et al. Distribuição espacial da tuberculose e a relação com condições de vida na área urbana do município de Campina Grande, 2004 a 2007. Revista Brasileira de Epidemiologia. 2012;15(1):222-232.

21. Scatena LM, Villa TCS, Netto AR, Kritski AL, Figueiredo TMRM, Vendramini SHF et al. Dificuldades de acesso a serviços de saúde para diagnóstico de tuberculose em municípios do Brasil. Revista de Saúde Pública. 2009;43(3):389-397. 
22. Bai J. Using spatial analysis to identify tuberculosis transmission and surveillance. In: Bai J. Computer Engineering and Networking. Switzerland: Springer; 2014. p. 337-344.

23. Roza DL. Padrões espaço-temporais da incidência da tuberculose em Ribeirão Preto, SP: uso de um modelo bayesiano auto-regressivo condicional. [Teses de Doutorado]. São Paulo: Faculdade de Saúde Pública, Universidade de São Paulo; 2011.

24. Souza WV, Ximenes R, Albuquerque MFM, Lapa TM, Portugal JL, Lima MLC, et al. The use of socioeconomic factors in mapping tuberculosis risk areas in a city of northeastern Brazil. Revista Panamericana de Salud Pública. 2000;8(6):403-410.

25. Souza WV, Albuquerque MFM, Barcellos CC, Ximenes RAA, Carvalho MS. Tuberculosis in Brazil: construction of a territorially based surveillance system. Revista de Saúde Pública. 2005;39(1):82-89.

26. Souza WV, Carvalho MS, Albuquerque MF, Barcellos CC, Ximenes RA. Tuberculosis in intra-urban settings: a bayesian approach. Tropical Medicine and International Health. 2007;12(3):323-330.

27. Zaragoza Bastida A, Hernández Tellez M, Bustamante Montes LP, Medina Torres I, Jaramillo Paniagua JN, Mendoza Martínez GD, Ramírez Durán N. Spatial and Temporal Distribution of Tuberculosis in the State of Mexico, Mexico. The Scientific World Journal. 2012;2012:570278. doi: $10.1100 / 2012 / 570278$.

28. Silva Jr JB. Diferenciais intra-urbanos de saude em Olinda/PE. [Dissertação de Mestrado]. São Paulo: Universidade Estadual de Campinas; 1995.

29. Xin Y, Xiao GS. Linear regression analysis: theory and computing. Hackensack: World Scientific; 2009.

30. Souza WV. O uso de informaçöes sócio-econômicas na construçäo de indicadores de situaçäo coletiva de risco para a ocorrência da tuberculose em Olinda, estado de Pernambuco. [Dissertação de Mestrado]. Recife: Centro de Pesquisas Aggeu Magalhães, Fundação Osvaldo Cruz; 1998.

31. Souza WV. A epidemiologia da tuberculose em uma cidade brasileira na última década do século XX: uma abordagem espacial. [Teses de Douturado]. Recife: Centro de Pesquisas Aggeu Magalhães, Fundação Osvaldo Cruz; 2003.

32. Instituto Brasileiro de Geografia e Estatística. Malha digital dos setores censitários urbanos do censo 2000: arquivos em formato Shapefile [Internet]. 2000 [citado 1 feb 2013]. Disponível em: https://tinyurl.com/y7cpsxfx.

33. Instituto Brasileiro de Geografia e Estatística. Malha digital dos setores censitários do censo 2010: Arquivos em formato Shapefile [Internet]. 2010 [citado 1 feb 2016]. Disponível em: https:// tinyurl.com/yaextjey

34. Greeland S. Medidas de efeito e associação. In: Rothman KJ, Greenland S, Lash TL. Epidemiologia moderna. Porto Alegre: Artmed; 2011. p. 67-88.

35. Tweya $\mathrm{H}$, Kanyerere, $\mathrm{H}$, Ben-Smith $\mathrm{A}$, Kwanjana J, Jahn A, Feldacker C, Gareta D, Mbetewa L, Kagoli M, Tikhalenawo Kalulu M, Weigel R, Phiri S, Edginton M. Re-treatment tuberculosis cases categorised as "other": are they properly managed? PLoS One. 2011;6(12):e28034. doi: 10.1371/journal.pone.0028034

36. Mak A, Thomas A, Del Granado M, Zaleskis R, Mouzafarova N, Menzies D. Influence of multidrug resistance on tuberculosis treatment outcomes with standardized regimens. American Journal of Respiratory and Critical Care Medicine. 2008;178(3):306-312.

37. Queiroz EM, De La Torre Ugarte-Guanilo MC, Ferreira KR, Bertolozzi MR. Tuberculose: limites e potencialidades do tratamento supervisionado. Revista Latino-Americana de Enfermagem. 2012;20 (2):369-377.

38. Monroe AA, Gonzales RIC, Sassaki CM, Netto AR, Villa TCS. Gerenciamento de caso ao doente/ família com tuberculose: uma estratégia de sistematização do cuidado no domicílio. Jornal Brasileiro de Pneumologia. 2005;31(1):91-92.

39. Maciel ELM, Vieira LWH, Molina LPD, Alves R, Prado TN, Dietze R. Comuni $\neg$ cantes domiciliares jovens de pacientes com TB pulmonar na região da grande Vitória (ES): um estudo de coorte. Jornal Brasileiro de Pneumologia. 2009;35(4):359366.

40. Gazetta CE, Ruffino-Netto A, Pinto Neto JM, Santos MLSG, Cury MRCO, Vendramini SHF et al. Controle de comunicantes de tuberculose no programa de controle da tuberculose em um município de médio porte da região sudeste do Brasil, em 2002. Jornal Brasileiro de Pneumologia. 2006;32(6):559-565.

41. Brasil, Ministério da Saúde, Secretaria de Vigilância em Saúde. Manual e recomendações para o controle da tuberculose no Brasil [Internet]. Brasília; 2011 [citado 1 feb 2016]. Disponible en: https:// tinyurl.com/y9js79ye. 
42. Pereira AGL. Distribuição espacial e contexto socioeconômico da tuberculose, Rio de Janeiro, Brasil. Revista de Saúde Pública. 2015;49:48.

43. Cantalice Filho JP, Boia MN, Sant`Anna CC. Análise do tratamento da tuberculose pulmonar em idosos de um hospital universitário do Rio de Janeiro, RJ, Brasil. Jornal Brasileiro de Pneumologia. 2007;33(6):691-698.

44. Mascarenhas MDM, Araújo LM, Gomes KRO. Epidemiological profile of tuberculosis among notified cases in the Municipality of Piripiri, Piauí State, Brazil. Epidemiologia e Serviços de Saúde. 2005;14(1):7-14.
45. Siqueira ASP. Determinantes socioeconômicos da produção da tuberculose: um estudo no município de Itaboraí, Região Metropolitana do Rio de Janeiro, no período de 2000 a 2011. [Teses de Doutorado]. Rio de Janeiro: Escola Nacional de Saúde Pública Sergio Arouca; 2014.

46. Muniyandi M, Rao VG, Bhat J, Yadav R, Sharma RK, Bhondeley MK. Health literacy on tuberculosis amongst vulnerable segment of population: special reference to Saharia tribe in central India. The Indian Journal of Medical Research. 2015;141(5):640-647.

\section{FORMA DE CITAR}

Silva APSC, Souza WV, Albuquerque MFPM. Factores asociados a la ocurrencia de tuberculosis y su distribución espacial en una ciudad brasileña, 1991-2010. Salud Colectiva. 2018;14(1):77-91. doi: 10.18294/sc.2018.1195

Recibido: 29 de octubre de 2016 | Versión final: 24 de abril de 2017 | Aprobado: 1 de junio de 2017

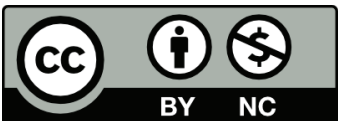

Este obra está bajo una licencia de Creative Commons Reconocimiento-NoComercial 4.0 Internacional. Reconocimiento - Permite copiar, distribuir y comunicar públicamente la obra. A cambio, se debe reconocer y citar al autor original. No Comercial — Esta obra no puede ser utilizada con finalidades comerciales, a menos que se obtenga el permiso.

http://dx.doi.org/10.18294/sc.2018.1195

Este artículo fue traducido del portugués por Viviana Martinovich. 